\title{
Empirical Validation in Agent-based Models: Introduction to the Special Issue
}

\author{
G. Fagiolo · C. Birchenhall · P. Windrum
}

Published online: 9 September 2007

(C) Springer Science+Business Media, LLC 2007

In the last two decades, economists have become more and more aware of the importance of modelling economies as complex dynamic systems (Rosser 2004). The recent tremendous improvement in computing capabilities has increasingly made it easier to implement computational simulation models in the study of markets, industries or organizations as evolving systems composed of many heterogeneous agents that exhibit bounded rationality and explicitly interact in a decentralized fashion. These models are known as agent-based models (ABMs) and the new field of research that has emerged is typically termed as agent-based computational economics (ACE). ${ }^{1}$

The extreme flexibility of ABMs in accommodating alternative modelling assumptions-including various forms of individual behaviour, interaction patterns and institutional arrangements - has allowed ACE researchers to explore the positive and

\footnotetext{
1 According to Tesfatsion, "ACE is the computational study of economic processes modelled as dynamic systems of interacting agents" (Tesfatsion 2006, p. 835). For the state of the art of ACE studies, see Tesfatsion and Judd (2006) and papers therein. See also Axtell (2000) and Pyka and Fagiolo (2007).
}

G. Fagiolo $(\varangle)$

Laboratory of Economics and Management, Sant'Anna School of Advanced Studies, Pisa, Italy

e-mail: giorgio.fagiolo@sssup.it

C. Birchenhall

University of Manchester, Manchester, UK

e-mail: chris.birchenhall@man.ac.uk

P. Windrum

Manchester Metropolition University Business School, Manchester, UK

e-mail: p.windrum@mmu.ac.uk

P. Windrum

MERIT, University of Maastricht, Maastricht, The Netherlands 
normative consequences of departing from the often over-simplifying assumptions characterizing most mainstream analytical models (Kirman 1997; Colander 2007). From the positive, descriptive side, ABMs have been successfully applied to a long list of both micro and macro issues including innovation and technological change; market dynamics; finance; industrial dynamics; organization theory; growth and development; international trade; macroeconomic dynamics and business cycles; evolution of institutions, social norms, and networks; political economy. ${ }^{2}$ More recently, ABMs have been applied to shed light in the area of economic policy and market design. ${ }^{3}$ Here the ACE approach is proving to be extremely fruitful. This is primarily because ABMs facilitate the construction and evaluation of policy measures in alternative institutional arrangements (market structure, auction types); behavioural and expectation-formation hypotheses for the agents involved (consumers, firms, government, unions, market traders); and interaction patterns (global versus local, social networks).

At the same time, research is increasingly focusing on the development of more sophisticated methods and protocols that can guide ACE researchers throughout the process that begins with the development and selection of an ABM (Edmonds and Moss 2005), goes on with the statistical analysis of its outputs (Pyka and Fagiolo 2007) and possibly ends with the evaluation of its policy implications (Moss 2002; Dawid and Fagiolo 2007). This research also aims to establish standards in ACE methodologies and, in particular, to improve comparability among different modelling exercises addressing similar questions (Leombruni et al. 2006).

A common theme informing both applied (positive, normative) analysis and methodological research in ACE concerns the relationships between ABMs and "real-world data". By real-world data we mean not only standard empirical evidence (e.g. datasets, stylized facts), but also qualitative and quantitative evidence regarding the setup of the economy and agents' cognitive repertoires gathered from laboratory experiments, case studies and inductive analyses. After all, one of the main justifications for using ABMs in economics, either as complements or substitutes of mainstream neoclassical models, relies in their capability to go beyond the constraints imposed by standard building blocks ${ }^{4}$ and to provide the micro-foundations based on "empirically sound" assumptions. At the same time, in order to compete with mainstream neoclassical models, ABMs have to confront empirical evidence and be better able to reproduce and explain existing observations. The interplay between ABMs and "real-world data" concerns all stages of model development and analysis and becomes crucial in assessing the success of ABMs within the community of researches, practitioners and end-users.

This special issue is devoted to explore the (often problematic) relationships between ABMs and "real-world data". In particular, the papers in this SI investigate open problems related to empirical validation of ABMs. The expression empirical valida-

\footnotetext{
2 For a comprehensive discussion and downloadable material on both ACE methodology and applications, the interested reader can refer to the website http://www.econ.iastate.edu/tesfatsi/ace, maintained by Leigh Tesfatsion.

3 Cf. the Special Issue on "Agent-Based Models for Economic Policy Design" edited by Herbert Dawid and Giorgio Fagiolo, forthcoming in the Journal of Economic Behavior and Organization.

4 Such as equilibrium analysis, full rationality and perfect foresight, representative individual hypotheses, and so on.
} 
tion (of an $\mathrm{ABM}$ ) typically means the procedure through which the modeller assesses the extent to which the model's outputs approximate reality, typically described by one or more 'stylized facts' drawn from empirical research. More generally, however, to "empirically validate" a given ABM can involve the appraisal of how "realistic" the set of model assumptions are (e.g. the behavioural rules employed by the agents in the model), or the evaluation of the impact of alternative market designs and/or policy measures.

As the papers in this special issue show, the debate on empirical validation of ABMs in economics is far from being settled and features many difficult-but very exciting-issues. Most of them are rooted in well-known, still open, problems in scientific methodology and concern empirical validation irrespective of the particular family of models under scrutiny. Others are strongly related to the peculiar ingredients that characterize ABMs. ${ }^{5}$

The discussion in the following papers also indicates that there is still a lot of heterogeneity among existing empirical-validation techniques. Some of these approaches stem from disciplines that have adapted ABMs in other areas (engineering, sociology, etc). ${ }^{6}$ However, economists and econometricians have recently begun to apply novel statistical techniques to empirically validate their ABMs. This SI presents applications of these new techniques and suggests interesting points for an "empirical agenda" of simulation models.

\section{The Papers in this Special Issue}

The first two papers of the special issue discuss methodological issues related to existing procedures of empirical validation in ABMs.

Fagiolo, Moneta and Windrum focus on micro-founded ABMs in economics. They claim that the existence of many, often conflicting empirical-validation techniques might be due to (at least) three reasons. First, as mentioned above, the relationship between real-world data and theoretical models is far from being straightforward, irrespective of the particular discipline within which this relationship is addressed. Second, comparability among ABMs is still difficult to perform, because of the existence of many alternative approaches to model selection and analysis. Finally, the very nature of ABMs naturally highlights deep methodological problems related to the comparison between statistical distributions of output traces (generated by complicated, often over-parameterized, stochastic processes) and single real-world observations. After critically surveying and contrasting the main approaches to empirical validation in ABMs, the paper offers a detailed discussion of (as yet) unresolved empirical-validation issues that require future research.

The paper by Brenner and Werker presents a novel taxonomy of ABMs in economics. They classify ABMs along two dimensions: the degree of generalization of the simulation model and the use of theoretical considerations versus empirical data. First,

\footnotetext{
5 For example: the presence of non-linear feedbacks between micro and macro layers; the often stochastic nature of behavioural rules; open-ended dynamics; direct interactions among agents; etc.

6 See, among others, Sargent (1998), Chattoe (2002) and Barreteau (2003). Cf. also Gilbert (2004).
} 
models can be general (respectively, specific) if the researcher analyses many (respectively, few) specifications of the simulation model. Second, the mix of real-world data and purely theoretical assumptions can favour the first ingredient (empirical models) or the second one (theoretical models). The authors survey the main classes of ABMs and classify them using these two dimensions. Furthermore, they single out the main steps that are conducted in developing and studying ABMs (building the model, conducting the runs, analyzing the results, etc.) and discuss their relative strengths and weaknesses. This enables them to better elucidate the relationships between real-world data and models. The authors offer the suggestion that researchers should prefer more general models and avoid as much as possible to impose restrictions arising from weak empirical evidence or ad hoc theoretical considerations. This position is complemented by the proposition that the only reliable results are those consistent with 'all simulation runs for all possible parameter sets'. The relationship of this position to statistical testing is not discussed. Finally, they identify and discuss a new type of simulation modelling approach (abductive inference), which, they claim, has a good potential to offer a solution for the trade-off between general-specific versus empirical-theoretical models and to better describe, explain and predict economic processes.

The remaining three papers focus instead on applications of empirical validation techniques and concepts to existing models and actual real-world data.

Bianchi et al. argue that in many ABMs the space of parameters is not sufficiently explored and results often refer to arbitrarily set parameter values. ${ }^{7}$ This is the case for the CATS model (Gallegati et al. 2005), which is an extremely successful ABM capable of replicating a wealth of micro and macro stylized facts. Bianchi et al. modify the setup of the original CATS model and perform extensive calibration and ex-post validation exercises using a database recording production and financial variables for a large sample of Italian firms over almost 20 years. The authors show that the CATS model, with parameters and micro initial conditions now being calibrated and estimated using real-world data, is still able to satisfactorily reproduce many stylized facts related e.g. the evolution of cross-section distributions of firm total capital and net worth. One notable feature of this paper is an introduction to indirect inference, ${ }^{8}$ which gives a clear bridge between ACE and mainstream econometric research agendas.

Understanding the reasons why ABMs are still far from being considered as a standard tool for economic analysis is one of the main themes of the paper by Marks. He argues that this may in part be due to two related factors. First, as discussed also in the paper by Fagiolo et al., ABMs often lack comparability. Whilst this seems to be true for most of ABMs in economics, Marks discusses how the "Energy Modeling Forum" at Stanford has been providing in the last 30 years a forum for comparison of simulation models addressing energy-related issues. Second, the high level of complexity of ABMs can make the process of empirical validation a very thorny problem. This is exacerbated because simulation models can prove existence but, contrary to closed-form analytical models, they can hardly demonstrate necessity. Marks presents a detailed overview of existing literature on empirical validation of simulation models

\footnotetext{
7 On this point, cf. also Fagiolo et al. (this special issue).

8 Cf. the work of Gourieroux and Monfort (1996) and Gilli and Winker (2003).
} 
and suggests a formal, operational framework for choosing among different methods of validation. The relationship between this framework and statistical hypothesis testing is not fully explored in this paper. Marks clarifies his views by using two examples: a simulation model developed to understand the complex interactions among consumers, retailers, and manufacturers in a market of competing coffee brands (Midgley et al. 2007); and the well-known Schelling's segregation model (Schelling 1971). Finally, Marks tackles the issue whether simulation models can deliver credible predictions. This is crucial in the case one simulates the evolution of market structure (e.g. merger decisions), which may be challenged in courts. It is interesting to notice that the extent to which courts can be convinced to trust simulation results strongly depends on the solution of the "counterfactual issue" (see Fagiolo et al., in this special issue, and Cowan and Foray 2002).

Finally, the paper by Sun and Tesfatsion focuses on a very exciting and promising area of application of empirically-validated ABMs. They report on the development and implementation of an ABM framework for testing the dynamic efficiency and reliability of the Wholesale Power Market Platform (WPMP, i.e. a market design proposed in 2003 by the U.S. Federal Energy Regulatory Commission for common adoption by all U.S. wholesale power markets and since then implemented in several U.S. areas). Due to the inherent complexity of its market design, however, the WPMP cannot be tested for reliability using standard analytical techniques. Therefore, Leigh Tesfatsion and her group have developed over the last years an ABM (called AMES) to computationally study the performance of the WPMP market design. As stated by the authors, AMES "models strategic traders interacting over time in a wholesale power market that is organized in accordance with core WPMP features and that operates over a realistically rendered transmission grid". Empirical validation in the AMES framework is carried out mainly at the "input" level. More specifically, empirical validity of "inputs" (i.e. market structure and architecture, traders behaviours, etc.) is ensured by means of an iterative participatory modelling approach (Barreteau 2003). In the words of Brenner and Werker (this special issue) this strategy allows the AMES to be an extremely specific and empirically-driven model. The authors are interested in using the AMES to investigate the performance of wholesale power markets operating under the WPMP market design, in terms of efficiency, profitability, and sustainability of existing generation and transmission facilities. The usefulness of the AMES framework is then illustrated in the paper by presenting a simple static five-node transmission grid test case. AMES provides an exemplar in its detailed specification and the use of open source software that opens up the door for replication and detailed verification by the ABM community.

Acknowledgements The Guest Editors would like to thank Hans Amman, Josephine Ruitenberg, Babylynne Andag and Esther Rentmeester, for their support throughout the whole process. Many thanks also to all the authors and referees for their collaboration, without which it would not have been possibile to produce this Special Issue so efficiently. 


\section{References}

Axtell, R. (2000). Why agents? On the varied motivation for agent computing in the social sciences. Working Paper No. 17, Center on Social and Economic Dynamics, The Brookings Institution.

Barreteau, O. (2003). Our companion modelling approach. Journal of Artificial Societies and Social Simulation, 6. available at http://jasss.soc.surrey.ac.uk/6/2/1.html

Chattoe, E. (2002). Building empirically plausible multi-agent systems: A case study of innovation diffusion. In K. Dautenhahn (Ed.), Socially intelligent agents: Creating relationships with computers and robots. Dordrecht: Kluwer.

Colander, D. (Ed.). (2007). Post-walrasian macroeconomics. Cambridge: Cambridge University Press.

Cowan, R., \& Foray, D. (2002). Evolutionary economics and the counterfactual threat: On the nature and role of counterfactual history as an empirical tool in economics. Journal of Evolutionary Economics, 12, 539-562.

Dawid, H., \& Fagiolo, G. (Eds.). (2007). Special issue on "Agent-Based Models for Economic Policy Design". Journal of Economic Behavior and Organization, forthcoming.

Edmonds, B., \& Moss, S. (2005). From KISS to KIDS - an 'anti-simplistic' modelling approach. In P. Davidsson, B. Logan, \& K. Takadama (Eds.), Multi agent based simulation 2004, Vol. 3415, (pp. 130-144) Lecture Notes in Artificial Intelligence, Springer.

Gallegati, M., Delli Gatti, D., Di Guilmi, C., Gaffeo, E., Giulioni, G., \& Palestrini, A. (2005). A new approach to business fluctuations: Heterogeneous interacting agents, scaling laws and financial fragility. Journal of Economic Behavior and Organization, 56, 489-512.

Gilbert, N. (2004). Open problems in using agent-based models in industrial and labor dynamics. In R. Leombruni, \& M. Richiardi (Eds.), Industry and labor dynamics: The agent-based computational approach (pp. 401-405). World Scientific: Singapore.

Gilli, M., \& Winker, P. (2003). A global optimization heuristic for estimating agent-based models. Computational Statistics and Data Analysis, 42, 299-312.

Gourieroux, C., \& Monfort, A. (1996). Simulation-based econometric methods. Oxford: Oxford University Press.

Kirman, A. (1997). The economy as an interactive system. In W. B. Arthur, S. N. Durlauf, \& D. Lane (Eds.), The economy as an evolving complex system II. Santa Fe: Addison-Wesley.

Leombruni, R., Richiardi, M., Saam, N. J., \& Sonnessa, M. (2006). A common protocol for agent-based social simulation. Journal of Artificial Societies and Social Simulation, 91. available at http://jasss.soc. surrey.ac.uk/9/1/15.html

Midgley, D. F., Marks, R. E., \& Kunchamwar, D. (2007). The building and assurance of agent-based models: An example and challenge to the field. Journal of Business Research, 60, 884-893.

Moss, S. (2002). Policy analysis from first principles. Proceedings of the National Academy of Sciences, 99, 7267-7274.

Pyka, A., \& Fagiolo, G. (2007). Agent-based modelling: A methodology for neo-schumpeterian economics. In H. Hanusch, \& A. Pyka (Eds.), The Elgar companion to neo-schumpeterian economics. Cheltenham: Edward Elgar.

Rosser, J. B. (Ed.). (2004). Complexity in economics. The International Library of Critical Writings in Economics, 174, Edward Elgar, Aldergate, 3 volumes.

Sargent, R. P. (1998). Verification and validation of simulation models. Proceedings of 1998 Winter Simulation Conference. California: San Diego.

Schelling, T. C. (1971). Dynamic models of segregation. Journal of Mathematical Psychology, 1, $143-186$.

Tesfatsion, L. (2006). ACE: A constructive approach to economic theory. In L. Tesfatsion, \& K. Judd (Eds.), Handbook of computational economics II: Agent-based computational economics. Amsterdam: North Holland.

Tesfatsion, L., \& Judd, K. (Eds.). (2006). Handbook of computational economics II: Agent-based computational economics. Amsterdam: North Holland. 